\title{
A randomized, double-blinded, controlled trial of herbal medicine combination for leg symptoms due to chronic venous disease
}

\author{
Anan Udombhornprabha ${ }^{1 \oplus}$, Naowarat Kanchanakhan ${ }^{2,}$, Pichai Phongmanjit $^{3}$
}

${ }^{1}$ Faculty of Pharmacy, the Western University - Bangkok Thailand

${ }^{2}$ College of Public Health Sciences, Chulalongkorn University, Bangkok Thailand

${ }^{3}$ Department of Surgery, Chiang Rai Prachanukroh Hospital, Chiang Rai, Thailand

\section{Correspondence}

Naowarat Kanchanakhan, College of Public Health Sciences, Chulalongkorn University, Bangkok Thailand

Email: naowarat.k@chula.ac.th

\section{History}

- Received: Jan 24, 2019

- Accepted: Feb 10, 2019

- Published: Feb 28, 2019

\section{DOI :}

https://doi.org/10.15419/bmrat.v6i2.523

\section{Check for updates}

\section{Copyright}

( ) Biomedpress. This is an openaccess article distributed under the terms of the Creative Commons Attribution 4.0 International license.

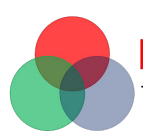

\begin{abstract}
Background: The herbal medicine combination (HMC) containing 2\% Asiaticoside and 1\% Acemannan, in a Beeswax encapsulation of $2 \%$ Acetylsalicylic acid base, was developed as a topical treatment option for mild-to-moderate chronic venous disease (CVD). For control (C), Beeswax encapsulation of 2\% Acetylsalicylic acid base was used. Methods: A double-blinded, randomized controlled trial comparing HMC with C was investigated among 42 CVD patients; 21 patients was allocated to receive either HMC or C, for a continuous 12-week treatment. To assess the efficacy, the following were employed: (i) Venous Clinical Severity Score (VCSS), (ii) Patients Self-Rating Symptoms Score (PSSS), and (iii) Short-form health-related quality of life score based on a Medical Outcomes Study 14-item Chronic Venous Disease (MOS CIVIQ14). The short-term safety was assessed by the patients' self-reported adverse events. The primary endpoint was a responder rate defined by a $50 \%$ reduction of the VCSS from baseline after 4 weeks of treatment. The secondary endpoints were assessed by improvement of overall disability after employing VCSS, PSSS, and MOS CIVIQ14, accordingly. Results: CVD Thai patients $(\mathrm{N}=42)$, regardless of clinical severity of CEAP class (CEAP1/CEAP2)or co-morbidity, responded 57.14\% to HMC $(\mathrm{N}=21)$ as compared with $9.52 \%$ to $C(N=21)$ the responder rate was significantly dependent on intervention $(p=0.003$ and $p=0.003$, respectively). HMC reduced VCSS markedly better than C, over the 4weeks: 11.9048 (6.4908) vs. 22.4702 (7.9438), $p<0.001$. HMC reduced VCSS and PSSS scores significantly better than C did: from baseline to 2 weeks ( $p=0.001$ vs. $p=0.017$, respectively), from 2 to 4 weeks ( $p=0.001$ vs. $p=0.004$, respectively), and from 8 to 12 weeks ( $p=0.001$ vs. $p=0.003$, respectively). After 12 weeks from baseline, from both the global and subscores for MOS CIVIQ14,treatment with HMC and C significantly improved scores $(p<0.001$ and $p<0.001$, respectively). For the adverse drug events reported, there were no statistically significantly differences between HMC and C from baseline to the end of the 12-week follow-up ( $p=0.756)$. Conclusion: The herbal medicine combination (HMC), consisting of $2 \%$ Asiaticoside and 1\%Acemannan in Beeswax encapsulation of 2\%Acetylsalicylic acid, as a topical administration in optional medical management for Thai patients with leg symptoms due to chronic venous disease, provided a clinical responder rate that was better than that of control. In terms of short-term safety assessments, no significant statistical differences for adverse events were reported with regards to incidence for both HMC and $C$ treatments. Further studies are warranted to explore and develop this herbal medicine base combination.
\end{abstract}

Trial registration no.: ISRCTN54360155

Key words: Acemannan, Acetylsalicylic acid, Asiaticoside, Chronic venous disease, Doubleblinded Randomized Control Trial, Herbal medicine combination, Leg symptoms

\section{INTRODUCTION}

Chronic venous disease (CVD) remains a global health burden, especially in the West, where is it reported that incidence of varicose veins is as high as $20-33 \%$ of the population. In most cases, clinical history and physical examinations that are used to assess the patient's severity of symptoms can help confirm the diagnosis of $\mathrm{CVD}^{1,2}$. In Thailand, a high incidence $(32.99 \%)$ of superficial varicose veins has been reported. However, these patients do not usually present with serious problematic leg symptoms. In addition, it has been reported that patients with probable CVD diagnosis often do not seek medical treatment, as in the case of female factory workers, particularly in Thailand ${ }^{3}$. Moreover, other important findings have been reported, such as that hospitalized leg ulcer patients in Thailand were mainly diagnosed with late-stage CVD with leg ulcers, primarily of venous origin, which often rise basically due to the untreated $\mathrm{CVD}^{4}$.

Nevertheless, these problematic leg symptoms reported by specialists in Thailand can vary differently among young and older CVD patients ${ }^{5}$. An epidemiological study (in the primary care setting) of patients diagnosed with probable chronic venous insuf- 
ficiency (CVI) reported that the patients experienced problematic leg symptoms (such as heaviness in the legs and itching) as their most common symptoms ${ }^{6}$. Though gross appearances of cutaneous skin lesions, such as varicose veins, were essential to confirm the diagnosis of CVD, other parameters (such as the concurrent presence of troubling symptoms) should also be helpful to secure CVD diagnosis ${ }^{7}$.

In practice, many patients with venous insufficiency do not receive proper early diagnosis and thereby early treatment ${ }^{8}$. Inadvertently, many of such silent CVD patients remain under-treated in some way, whereby patients still seek symptomatic treatment with other systematic drugs, especially various types of painkillers. This was basically due to the unavailability of effective standard medications for the treatment of CVD. The current treatment modalities have primarily been the use of compression stocking and encouragement of patients to make lifestyle modifications.

Since there has been no effective standard medications for the management of CVD, attempts have been initiated to develop medications, especially herbalbased medicines, that are safe and effective to alleviate overall problematic leg symptoms of CVD. Subsequently, the literature reviews for the possible selection of potential safe and effective herbal-based medicinal agents were performed through electronic database searching via PubMed, Medline, Google Scholar, and the Cochrane Library. The search query began with "drug treatment of chronic venous disease" filtered further by tested in a clinical trial, available as at least abstracts, and investigation only in humans from 1960 to 2015 . There were only 356 articles found. Subsequently, an exhaustive screen was performed through each of the abstracts to exclude the overlapping CVI reported within either the domain of other cardiovascular diseases or CVI mimics with venous leg ulcers. Furthermore, the literature search was subjected to data exclusion and agreements among researchers regarding the searched files, and consultation with expert vascular specialist was conducted for their opinions. Despite reported studies already showing efficacy and safety of the herbal drugs, there have also been some conflicting evidence of the actual efficacy ${ }^{8-36}$. This was due mainly to inconclusive or inexplicable mechanisms of action, which were likely a result of limitations of the study design.

Among the active herbal medicinal agents, two major active herbal extracts, namely (1) Titrated Extract Centella Asiatica (TECA) - Asiaticoside, and
(2) Acemannan (Aloe vera) were identified. In addition, Acetylsalicylic Acid (ASA) base was added to the above to enhance skin absorption via Beeswax encapsulation, thereby rendering greater therapeutics effects. These selections were based on the possible synergy of different and combined mechanisms of action, such as anti-inflammation, anti-fibrosis, anticoagulation, antibacterial activity, and immunomodulation $^{9-42}$.The two herbal-medicine combinations were obtained through the USFDA-verified global commercial source of herbal plant extracts, to ensure the identity of the compounds in the herbal medicine, and ensure that the semi-purified extracts were of known content and precise formulation. The herbal medicine combination (HMC) of the topical formulation was formulated and produced to adequate levels to ensure sufficiency for a clinical trial. This was conducted by incorporation of the two herbal medicine extracts with known contents with the Acetylsalicylic acid encapsulation base. Later, a research protocol for both the Institution Review Board and the Hospital Ethical Approval Committee were granted. The investigation clinical trial was thereby registered within the BioMed central domain as ISRCTN No.54360155.

\section{METHODS}

\section{Preparation of Herbal Medicine Combina- tion (HMC)}

The preparation of HMC was done according to standard preparation procedures for pharmaceutical formulation. These procedures included:

(1) acquisition of standard raw material of reliable standard herbal extract sources,

(2) preparation of gel-based containing Acetylsalicylic Acid microencapsulation pre-formulation,

(3) preparation of finished herbal medicine combination as a test product containing AcemannanAsiaticoside as an active treatment and an identical control treatment without Acemannan-Asiaticoside,

(4) analysis of absorption using Franz cell diffusion studies based on Acetylsalicylic acid encapsulation, and

(5) microbial analysis of Acemannan-Asiaticoside test product and a control product.

All pre-formulations, formulations, and production processes were conducted at the pharmaceutical laboratories of the Eastern Asia University School of Pharmacy, and duly kept as internal files to be published in relevant publications. 


\section{Aim}

The aim was test the hypothesis that the response to treatment of mild-to-moderate CVD, with topical application of an HMC containing 2\% Asiaticoside and $1 \%$ Acemannan (HMC), in a Beeswax encapsulation of $2 \%$ Acetylsalicylic acid base, would provide $35 \%$ better response, as compared with the Beeswax encapsulation of $2 \%$ Acetylsalicylic acid base alone (control: C).

\section{Design of the study}

The study population consisted of Thai patients diagnosed with CVD based on CEAP Classification. The study sample consisted of CVD patients diagnosed by the surgeon as CEAP class 1 to 4 , and recruited form Somdet Prayanna Sangworn Chiang Rai and/or Chiang Rai Hospital. These patients were ambulatory patients who fulfilled the eligibility requirement and conformed to the inclusion and exclusion criteria. The prospective patients were consecutively recruited as per eligibility/inclusion and exclusion criteria, interviewed, and screened; those participating in the study provided signed consent forms. All patients were diagnosed as CVD and classified as CEAP 1 to 4 by a surgeon. The research design was an interventional, prospective, randomized and double-blind, controlled trial. Patients were prospectively recruited and the interventions were randomly allocatedin a concealment aluminum tube either with HMC or C. For double-blinding of the patients, nurses and surgeon received a computer-generated set of two randomized numbers for the patients through consecutive randomization. All patients who were recruited into the study fulfilled the inclusion/exclusion criteria. Patients seeking treatment and medical advice at the surgery ambulatory clinics were recruited since they fulfilled at least two of the following leg symptomsas follows: Heavy legs, Pain in the legs, Sensation of leg swelling, Leg cramping at night, Itching legs - or Pin in the leg, Sensation of burning, Sensation of severe pins/needle in the leg, Presence of superficial varicose vein, Presence of spider veins, and Presence of blood-clotting. All patients had been accurately diagnosed as CVD and were graded as the appropriate CVI stage per the CEAP Criteria by the surgeon. The non-inclusion/exclusion criteria included patients with known history of Acetylsalicylic acid (ASA)-exacerbated respiratory disease (AERD) and/or asthma, ASA-sensitivity and Rhinitis/Nasal Polyps, ASA- and NSAIDs-induced cutaneous reactions with Urticaria, Angioedema, ASA-induced anaphylactoid reactions with Hypotension, swelling, laryngeal edema, generalized pruritus, and/or tachypnea, as well as those with known sensitivity or allergy to Titrated Extracts of Centenella Asiatica (TECA) or Asiaticoside, and/or known sensitivity or allergy to Acemannan and aloe vera products.

\section{Intervention}

Participants fulfilling the eligibility criteria were randomly allocated into one of two arms:

(1) the active treatment arm (each of the participants was given HMC and instructed to apply it to the symptomatic leg once in the morning and again at bedtime continuously for 12 weeks), and

(2) the control treatment arm (each of the participants was given $\mathrm{C}$ with the same odor and color as HMC with no active herbal drugs). All patients were instructed to apply $10-15 \mathrm{~mL}$ of the test product (concealed in the aluminum tube provided) onto the symptomatic leg once in the morning and again at bedtime continuously for 12 weeks. All other medications, including oral medications, were not allowed during the trial period. At each of the early weekly visits, patients were provided 2 aluminum tubes of the test products, and thereafter 8 tubes for each of the next four weeks of the visit. At each of the follow-up visits, patients were instructed to return the test products.

\section{Outcome measures (Dependent Outcomes)}

\section{Primary outcome measure}

The primary outcome was responder rate (\%), which reflects the venous disease severity, as assessed by $50 \%$ score reduction from baseline of the venous clinical severity score (VCSS) ${ }^{38,39}$. This was measured after 4weeks and adjusted with co-morbidity and CEAP severity ${ }^{40}$.

\section{Secondary outcome measure}

The secondary outcome was disability caused by venous disease as assessed by venous clinical severities via:

(1) VCSS (after 4 and 8 weeks of treatment, and at the end (12 weeks) of treatment),

(2) Disease-specific symptoms (adapted for Patient Self-Rated Symptoms Perception Score (PSSS) as visual analog scale ${ }^{41}$, measured at baseline, after 1,2,3,4, and 8 weeks, and at the end ( 12 weeks) of treatment), (3) Medical Outcomes Study 14-item Short Form Health Survey for Chronic Venous Insufficiency (MOS CIVIQ14), which was adapted as a Thai ver$\operatorname{sion}^{42}$, and used for evaluation at baseline and after the end (12 weeks) of treatment, and

(4) Adverse incidental events as reported by patients in a weekly diary follow-up (subjective defined rate). 
Sample size determination and statistical analysis

\section{Sample size calculation}

The estimated response to active treatment was based on $50 \%$ reduction of the objective score of Venous Clinical Severity Score (VCSS) from baseline, and is evaluated by a physician and compared with a control treatment. As such, the sample size was computed based on the responder rate (\%) for 2 independent proportions of the 2 different treatment groups. The sample size estimation was based on the primary objective in improvement of responder rate for active treatment as compared with controlled treatment (Control), as two independent proportions after 4 weeks of treatment. Responder patients were assessed with at least $50 \%$ score reduction from the baseline score after 4 weeks of treatment. This sample size estimation was, therefore, based on one sample test of 2 independent proportions to show the superiority of responders to treatment. Based on the hypothesis, the normal distribution for the probabilityof responders was assessed, as was analysis of differences in the 2 independent proportions.

The primary efficacy endpoint was responder rate after 4 weeks of treatment with the Null hypothesis that both groups reflected no differences in responder rate, and the Alternative Hypothesis with a 2-sided test, alpha $=0.05$, at $80 \%$ power to detect the differences. Based on a balanced controlled design, especially including placebo and drug effects, where a possible placebo and drug response could be found in at least $10 \%$ responders, whereas the responder rate of the active treatment should be $35 \%$ or greater than the control. The formula for calculating significant differences was based on the Chi-Square test. We assumed the continuity correction and possible $10 \%$ dropout, thereby deriving an optimal total sample size of 47 patients. Eligible participants with leg symptoms or complaints and who were seeking advice at ambulatory surgery clinics were informed about the study by two research nurses.

The participant recruitment began on November $1^{s t}$, 2015 , for a period of 3 months. A total of 66 participants were obtained in the beginning. Once participants agreed to participate, they were explained in further detail about the study, and were independently interviewed and informed about study procedures before being referred to the surgeon for physical examination, diagnosis, and CEAP grading of CVD prior to inclusion in the study. Only diagnosed and graded CVD patients were included in the study. Patients included in the study signed informed consent forms, and were referred to concealed treatment allocation and appointments for followup by two research nurses. Patient sampling was conducted by consecutive random sampling andconcealed treatment allocations were given as blinded randomizations; patients either received treatment 1 (HMC) or treatment 2(C). The diagnosed CVD patients recruited to this investigation were given inpatient screening flowcharts, as shown in Figure 1.

A specified censured data collection for each reason for withdrawal from study could be generated. There were unobserved sample data mainly due to loss of follow-up early on or at the beginning of the recruitment phase during pre-enrollment; as such, the censure method was employed for this investigation for the unobserved samples as a random loss due to lack of follow up. This censure method should be justified in dealing with handling reasons and timing of withdrawals. Moreover, it can consequently avoid the possible relationships between missing data and the outcomes of interest. The analysis of this investigation was, therefore, conducted and based on a per protocol analysis.

\section{Statistical analysis}

Analysis of the descriptive statistics was employed with hypothesis testing. The analysis was based on the assumption that active treatment should provide $35 \%$ or greater responder rate than control treatment, whereby responder rate was defined by the standardized Venous Clinical Severity Score (VCSS) as a transformed percentage score, whereby there should be a $50 \%$ score reduction from baseline after 4 weeks of treatment as the response to treatment. The overall statistical analysis was performed for primary and secondary outcomes with input variable as nominal (HMC or C) or various independent variables, and output variable as outcomes or dependent variables (ratio, quantitative discrete or quantitative normal), or as Chi-square test ( $\chi^{2}$-test) for responder rate between active treatment (HMC) vs. controlled treatment (C), or as primary outcomes for categorical outcomes (ratio) for one sample of two independent groups. The t-test analysis (assuming a normal distribution) for comparison of mean score differences within treatment (HMC or C) at baseline, weeks 4, 8, and 12 for VCSS, and PSSS total score as secondary outcomes. The paired t-test and t-testanalysis were performed for each count after treatment and comparisons were made between active treatment (HMC) and control treatment (C). Then, the domain 


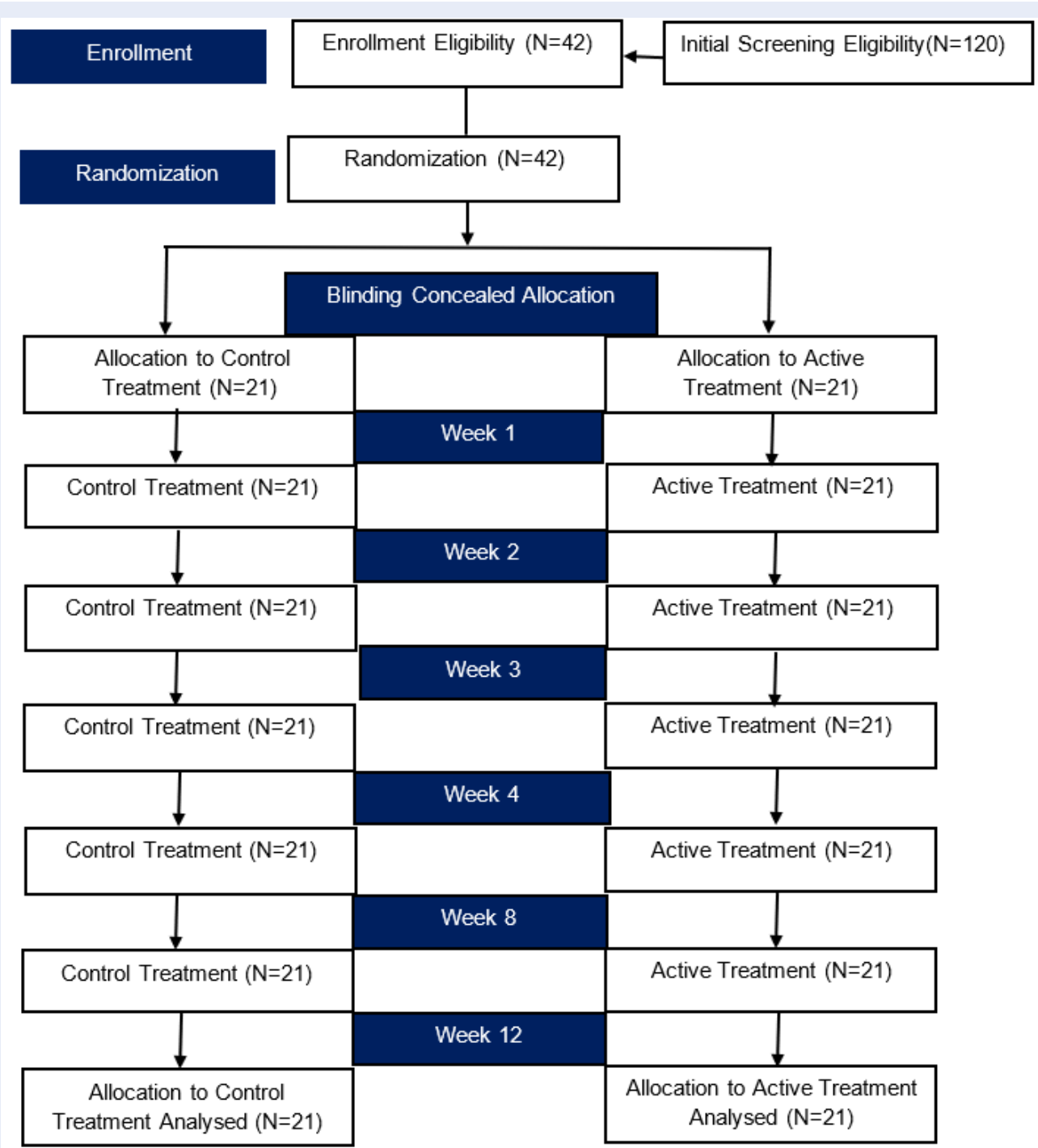

Figure 1: Patient screening flow-chart. Note. 1. Consecutive randomization; 2. Test products allocation were blinding $\mathrm{b} / \mathrm{w}$ physician-nurse-patients; $\mathbf{3}$. Each of any one of two set of test products contained matching computerized allocated randomization numbers as series on products.

score and subscore of MOS CIVIQ14, before and after HMC, were analyzed with paired t-test. The Fisher's Exact Probability Test for total spontaneous adverse events were reported and compared between the two groups, with non-parametric statistics as secondary outcomes (quantitative discrete). The analysis assumed $80 \%$ power and with significance level at $\mathrm{p}<0.05$.

\section{RESULTS}

A different pre-specified censure data for each different reason for withdrawal could be anticipated. There were unobserved sample data mainly due to loss of follow-up at an early stage of the recruitment, such as before the investigation at the pre-enrollment. A censure method was employed for the unobserved samples as a loss of follow up. We believe this covers various reasons such as timing of withdrawals. The consequent potential relationship between missing data and the responder rate could be avoided. The analysis of this investigation was performed based on a per protocol analysis, and included all recruited patients per protocol. The baseline patient demographic and clinical characteristics are provided in Table 1. The overall leg symptoms for the Active (HMC) and the Control (C) groups are shown in Table 2. 


\begin{tabular}{|c|c|c|c|}
\hline Clinical Characteristics & $\begin{array}{l}\mathrm{C}(\mathrm{N}=21) \\
\text { Mean (SD), } \\
\text { Min-Max, Range }\end{array}$ & $\begin{array}{l}\text { HMC }(\mathrm{N}=21) \\
\text { Mean (SD), } \\
\text { Min-Max, Range }\end{array}$ & p-Value \\
\hline Gender Female & 21 & 21 & \\
\hline Age (in years) & $\begin{array}{l}48.90(6.09) \\
39-62,23\end{array}$ & $\begin{array}{l}47.00(5.42) \\
39-58,19\end{array}$ & 0.291 \\
\hline Weight (in Kg) & $\begin{array}{l}59.00(6.05) \\
48-68,20\end{array}$ & $\begin{array}{l}60.81(5.97) \\
49-74,25\end{array}$ & 0.336 \\
\hline Height (in CM) & $\begin{array}{l}159.28(6.93) \\
150-150,2\end{array}$ & $\begin{array}{l}160.23(5.94) \\
149-170,21\end{array}$ & 0.636 \\
\hline Family History of CVD & Yes (0), No (21) & Yes $(0)$, No $(21)$ & - \\
\hline Medical History with- & & & ${ }^{*} 0.191$ \\
\hline Comorbidity & $16(76.2 \%)$ & $17(80.1 \%)$ & \\
\hline None & $5(24.2 \%)$ & $2(9.7 \%)$ & \\
\hline Hypertension & $0(0 \%)$ & $2(9.7 \%)$ & \\
\hline Musculoskeletal & $0(0 \%)$ & $0(0 \%)$ & \\
\hline Self-Medication (OTC) & & & ${ }^{*} 0.707$ \\
\hline Sometime & $17(82.5 \%)$ & $16(77.8 \%)$ & \\
\hline Regular & $4(17.5 \%)$ & $5(22.3 \%)$ & \\
\hline CEAP Severity Classification & & & ${ }^{*} 0.533$ \\
\hline CEAP C1 & $8(38.0 \%)$ & $10(47.6 \%)$ & \\
\hline CEAP C2 & $13(62.0 \%)$ & $11(52.4 \%)$ & \\
\hline SBP (mmHg) & $\begin{array}{l}138.95(4.44) \\
129.0-146.0,17.0\end{array}$ & $\begin{array}{l}137.86(4.06) \\
130.0-145.0,15.0\end{array}$ & 0.410 \\
\hline DBP (mmHg) & $\begin{array}{l}88.04(3.90) \\
78.0-97.0,19.0\end{array}$ & $\begin{array}{l}88.57(2.54) \\
83.0-92.0,9.0\end{array}$ & 0.609 \\
\hline Heart Rate (tpm) & $\begin{array}{l}70.81(4.08) \\
64.0-78.0,14.0\end{array}$ & $\begin{array}{l}70.33(4.68) \\
60.0-78.0,18.0\end{array}$ & 0.727 \\
\hline Numbers of Leg Symptoms & $\begin{array}{l}3.71(0.902) \\
3-6,3\end{array}$ & $\begin{array}{l}3.05(0.669) \\
2-4,2\end{array}$ & ${ }^{*} 0.087$ \\
\hline
\end{tabular}

P-value by T-test, ${ }^{\star P}$-value by Chi-Square Test

Note: tpm: time per minute, CEAP: CVD can be classified orgraded as per the descriptive Clinical, Etiologic, Anatomical, and Pathophysiological (CEAP) classification ${ }^{40}$, CVD: Chronic Venous Disease, OTC: Over-The-Counter; Control: C, Active: Herbal Medicine Combination (HMC)

\section{Venous Clinical Severity Score Responder Rate}

Venous clinical severity score (VCSS) was graded independently by a surgeon subsequent to physical examination after a 4-week follow-up. The assessment confirmed that the Active treatment provided a responder rate of 12 out of 21 patients $(57.14 \%)$; this was a significantly better response than that of the Control, in which 2 out of 21 patients (9.52\%) responded $=0.003$ for comparison of the two groups. The re- sponder rate remained better for the Active treatment group after 8 and 12 weeks, versus the Control group: the data for which patients treated with the control group was 3 patients out of $21(14.28 \%)$ at $=0.009$, and 4 patients out of $21(19.04 \%)$ at $=0.025$, respectively (Table 3 ). 
Table 2: Leg symptoms of chronic venous disease patients treated with herbal medicine combination (HMC) and control (C)

\begin{tabular}{|c|c|c|c|}
\hline Leg symptoms & $\begin{array}{l}\mathrm{C}(\mathrm{N}=21) \\
\text { Number }(\%)\end{array}$ & $\begin{array}{l}\text { HMC (N=21) } \\
\text { Number (\%) }\end{array}$ & p-Value \\
\hline Heavy Leg & & & ${ }^{*} 0.525$ \\
\hline Yes & $7(43.7 \%)$ & $9(56.3 \%)$ & \\
\hline No & $14(53.8 \%)$ & $12(46.2 \%)$ & \\
\hline Leg Swelling & & & ${ }^{\star} 0.334$ \\
\hline Yes & $6(56.7 \%)$ & $9(43.3 \%)$ & \\
\hline No & $15(40.0 \%)$ & $12(60.0 \%)$ & \\
\hline Leg Pain & & & - \\
\hline Yes & $21(50.0 \%)$ & $21(50.05)$ & \\
\hline No & $0(0 \%)$ & $0(0 \%)$ & \\
\hline Night Cramp & & & ${ }^{\star} 0.121$ \\
\hline Yes & $12(57.1 \%)$ & $7(33.3 \%)$ & \\
\hline No & $9(42.9 \%)$ & $14(66.7 \%)$ & \\
\hline Burning Sensation & & & - \\
\hline Yes & $0(0 \%)$ & $0(0 \%)$ & \\
\hline No & $21(100 \%)$ & $21(100 \%)$ & \\
\hline Pin/Itching in Leg & & & ${ }^{\star} 0.050$ \\
\hline Yes & $1(4.8 \%)$ & $0(0 \%)$ & \\
\hline No & $20(95.2 \%)$ & $21(100.0 \%)$ & \\
\hline Leg Itching & & & ${ }^{\star} 0.311$ \\
\hline Yes & $10(47.6 \%)$ & $4(19.1 \%)$ & \\
\hline No & $11(52.4 \%)$ & $17(80.9 \%)$ & \\
\hline Varicose Vein & & & ${ }^{\star} 0.019$ \\
\hline Yes & $18(85.7 \%)$ & $11(52.4 \%)$ & \\
\hline No & $3(14.3 \%)$ & $10(47.6 \%)$ & \\
\hline Spider Veins & & & - \\
\hline Yes & $3(50.0 \%)$ & $3(50.0 \%)$ & \\
\hline No & $18(50.0 \%)$ & $18(50.0 \%)$ & \\
\hline
\end{tabular}

${ }^{*}$ P-value by Chi-Square Test.

Note: Control: C, Active: Herbal Medicine Combination (HMC) 


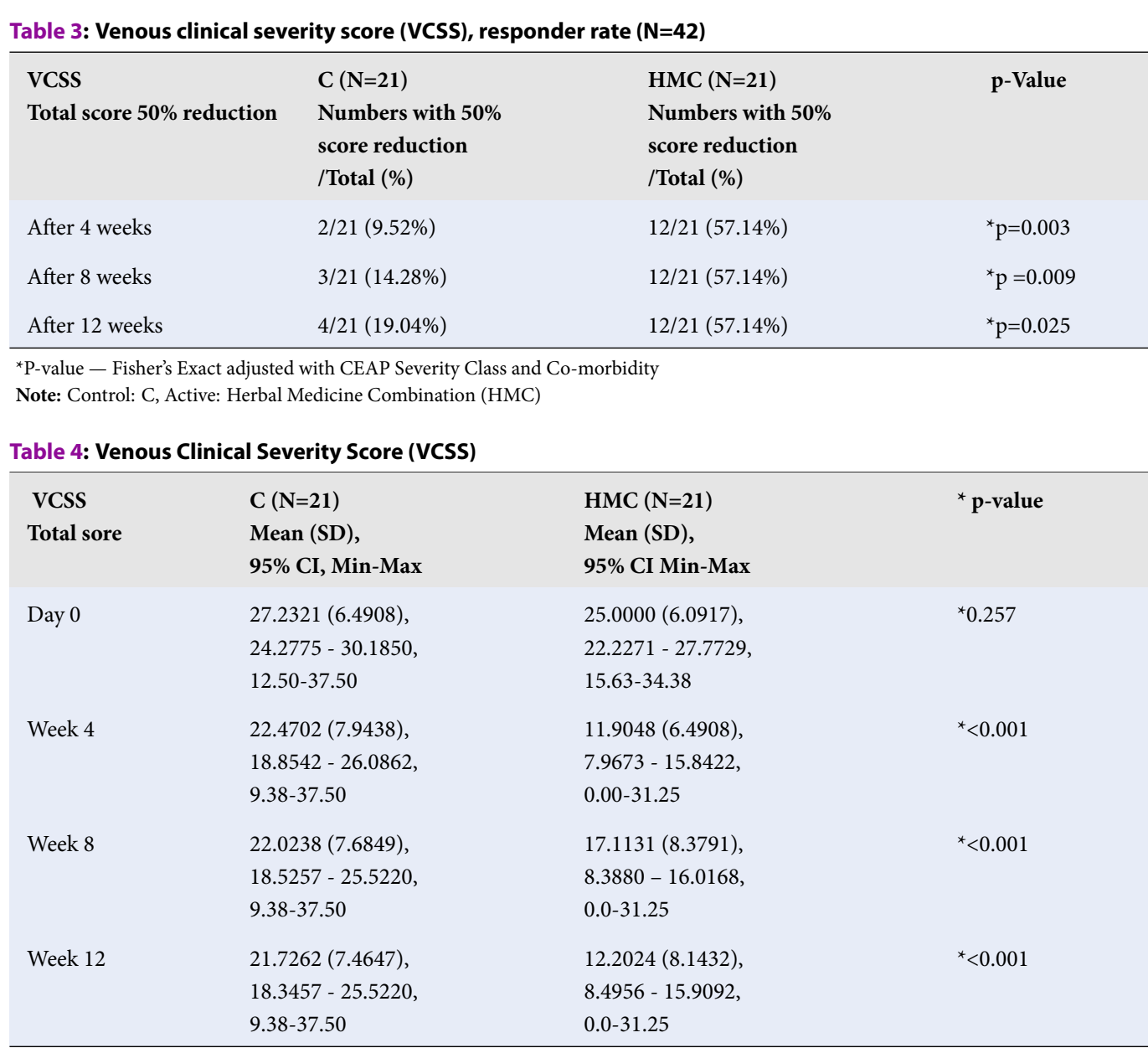

* Total Score

${ }^{*}$ P-value T-test; Note: Control $=\mathrm{C}$, Active $=$ Herbal Medicine Combination $($ HMC $)$

Table 5: Patient self-rated symptom perception score (PSSS) based on visual analog scale

\begin{tabular}{|c|c|c|c|}
\hline \multirow[t]{2}{*}{ Total score } & $C(N=21)$ & HMC (N=21) & ${ }^{\star}$ p-value \\
\hline & Mean (SD), 95\% CI, Min-Max & Mean (SD), 95\% CI, Min-Max & \\
\hline \multirow[t]{2}{*}{ Day 0} & $35.2585(8.0086)$ & $31.7211(6.2347)$ & 0.118 \\
\hline & $27.2499-43.2671,30.86-50.57$ & $25.4864-37.9558,26.71-38.57$ & \\
\hline \multirow[t]{2}{*}{ Week 1} & $24.8980(7.4151)$ & $19.2449(5.0210)$ & 0.006 \\
\hline & $17.4831-32.4041,26.71-38.57$ & $14.2239-24.2659,12.14-31.14$ & \\
\hline \multirow[t]{2}{*}{ Week 2} & 23.0680 (7.7269), & $17.1020(4.8117)$ & 0.005 \\
\hline & $15.3411-30.7949,26.86-37.29$ & $12.2903-21.9137,17.57-28.29$ & \\
\hline \multirow[t]{2}{*}{ Week 3} & $23.0816(7.7273)$ & $16.9864(5.0209)$, & 0.004 \\
\hline & $15.3543-30.8089,26.86-37.14$ & $23.4346-31.1686,19.57-29.43$ & \\
\hline \multirow[t]{2}{*}{ Week 4} & $22.9524(7.7321)$ & 16.8639 (4.9864), & 0.004 \\
\hline & $15.2203-30.6845,26.86-36.86$ & $11.9655-21.8503,19.57-29.43$ & \\
\hline \multirow[t]{2}{*}{ Week 8} & $22.9592(7.6331)$ & 16.7347 (4.7568), & 0.003 \\
\hline & $15.3261-30.5923,27.57-36.86$ & $11.9779-21.4915,18.57-28.14$ & \\
\hline \multirow[t]{2}{*}{ Week 12} & $22.8571(7.5951)$ & $16.6803(4.6080)$, & 0.003 \\
\hline & $15.2620-30.4522,25.86-35.86$ & $12.0723-21.2883,19.00-29.00$ & \\
\hline
\end{tabular}

${ }^{\star}$ Total Score

${ }^{*}$ P-Value T-test. Note: C: Control Treatment, HMC: Active Treatment 
Disability caused by venous disease: Venous Clinical SeverityScore

It was found that the baseline Venous Clinical Severity Score (total) between the control and active treatment groups was not different $(p=0.257)$. Subsequent to follow-up assessment for treatment effects of HMC $v s$. $\mathrm{C}$, the patients treated with Active treatment tended to have better improvement as assessed by venous clinical severity score after 4 weeks $(\mathrm{p}<0.001), 8$ weeks $(\mathrm{p}<0.001)$, and 12 weeks $(\mathrm{p}<0.001)$. They were tested by T-Test (assumption of normal distribution); comparing $\mathrm{HMC} v s$. C, we found that HMC treatment was better for improving overall symptoms (as assessed by reduction of venous severity clinical score at various periods). In this respect, the HMC treatments clearly provided better outcomes or improvement of disability, in terms of reduction of clinical severity as assessed by Venous Clinical Severity Score (Table 4).

\section{Disability caused by venous disease: $\mathrm{Pa}$ - tient Self-Rating Symptom Perception Score}

The patients also self-reported their treatment response by use of visual analog scale (VAS) assessment and Patient Self-Rating Symptom Perception Score (PSSS) which observed the total symptom score evolution from baseline.The PSSS score between HMC and $\mathrm{C}$ treatment at baseline did not show a statistically significant difference ( $\mathrm{p}=0.118$ ). However, subsequent to follow-up, in the assessment of the treatment effects between $\mathrm{C}$ and HMC, patients treated with HMC provided better improvement as assessed by PSSS reduction after 1 week $(p=0.006), 2$ weeks $(\mathrm{p}=0.005), 3$ weeks $(\mathrm{p}=0.004), 4$ weeks $(\mathrm{p}=0.004), 8$ weeks ( $p=0.003$ ), and at the end ( 12 weeks) of the trial $(p=0.003)$. These results were significantly different as compared with $\mathrm{C}$ treatment. In this regard, HMCtreatment provided better outcomes in terms of symptom improvement or improvement of disability with self-assessment by PSSS (Table 5).

Medical Outcomes Study 14-item Short Form Health Survey for Chronic Venous Insufficiency (MOS CIVIQ14)

The patients also self-reported their health-related quality of life; this was assessed by the Medical Outcomes Study 14-item Short Form Health Survey for Chronic Venous Insufficiency (MOS CIVIQ14) which was adapted into a Thai version. Theoriginal CIVIQ14 was first developed by Launois et al. ${ }^{42,43}$. The CIVIQ14 was a 14-item self-rated health survey questionnaire which covered questions in three health dimensions: Physical (7-item), Pain (1-item), and Psychological (6-item). The CIVIQ14 scoring was based on five response choices. The original CIVIQ14 Thai version was a validated official translation by a Thai vascular specialist. It was found that the health-related quality of life assessed by the MOS CIVIQ14 score for HMC treatment was statistically significant and improved the pain score from baseline with mean (SD), min-max and range of 63.5714 (9.3732), 45.0-75.0, and 30.0, respectively, after 12 weeks of treatment. The MOS CIVIQ14 sub-score was improved for Pain-score $(\mathrm{p}<0.001)$, Physical Function-Score $(\mathrm{p}<0.001)$, Psychological and Social Function-score $(p<0.001)$, and Global-score $(p<0.001)$. In these respects, HMC treatment therefore provided better outcomes and overall improvement in terms of health-related quality of life as self-assessed by the MOS CIVIQ14 (Table 6).

\section{Adverse events reported}

This investigation utilized what patients indicated as adverse incidents in their diary reports as the subjective definition of adverse events. The reports indicated the frequency of adverse events since reported adverse events for the pilot test of the formulation of both the HMC and C had been conducted earlier and had found no statistical significant localized skin lesions $^{44}$. This investigation implicated for the efficacy test of the same at the defined sample not the objective of spontaneous reporting of the adverse events. From the pilot test, it was found that there is a need for formulation revamping despite the fact that adverse events reported were similar between the HMC and $\mathrm{C}$ treatments. As such, reporting of adverse events in this investigation was restricted to local skin reaction and thereby could be followed through completion of the trial (at 12 weeks). As a result, our designated and convenient definitions were as follows: No (indicating no event), Some-tolerable (indicating some adverse events but patients could tolerate), High-intolerable (indicating serious adverse events and patients could not tolerate), and Fatal (indicating very serious adverse events, need to be investigated, and stop treatment immediately). In this regard, this investigation found that for the overall period of treatment, both HMC and C reflected no statistically significant differences in terms of adverse event frequency, from baseline to end of week- 4 ( $\mathrm{p}=0.628)$, week- 8 ( $\mathrm{p}$ $=0.376)$, or week-12 $(\mathrm{p}=0.756)$, as demonstrated in Table 7. 
Table 6: Medical outcomes study 14-item short form health survey for chronic venous insufficiency (MOS CIVIQ14) adapted Thai version

\begin{tabular}{|c|c|c|c|}
\hline MOS CIVIQ14 & $\begin{array}{l}\text { Baseline - Day } 0 \\
\text { Mean (SD), Min-Max, Range }\end{array}$ & $\begin{array}{l}\text { End of Trial/after Week } 12 \\
\text { Mean (SD), Min-Max, Range }\end{array}$ & p-value \\
\hline Pain ${ }^{*}$ & $\begin{array}{l}63.5714(9.3732) \\
45.00-75.00,30.00\end{array}$ & $\begin{array}{l}70.7143(6.9436) \\
60.00-75.00,15.00\end{array}$ & $<0.001$ \\
\hline **Physical Function & $\begin{array}{l}56.4286(5.4772) \\
49.29-66.43,17.14\end{array}$ & $\begin{array}{l}66.9388(3.0232) \\
64.29-75.00,10.71\end{array}$ & $<0.001$ \\
\hline $\begin{array}{l}\text { **} \text { Psychological \& Social } \\
\text { Function }\end{array}$ & $\begin{array}{l}61.3095(5.7347) \\
52.50-75.22,5.00\end{array}$ & $\begin{array}{l}70.4762(2.6947) \\
65.00-75.00,10.00\end{array}$ & $<0.001$ \\
\hline${ }^{* * * *}$ Global Score & $\begin{array}{l}70.4762(2.6947) \\
65.00-75.00,10.00\end{array}$ & $\begin{array}{l}78.70(5.8000) \\
76.06-81.34\end{array}$ & $<0.001$ \\
\hline
\end{tabular}

${ }^{*}$ P-value Paired T-Test.

Note: ${ }^{\star}$ Pain Score, ${ }^{* *}$ Physical Function Score, ${ }^{* *}$ Psychological-Social Function, ${ }^{* * *}$ Global Score (HMC Treatment at the end of trial- Baseline)

Table 7: Report of adverse events over 12-weeks period of follow-up

\begin{tabular}{|c|c|c|c|}
\hline Summary period & $\begin{array}{l}\text { Control }(\mathrm{N}=21) \\
\text { Number, }(\%)\end{array}$ & $\begin{array}{l}\text { Active }(\mathrm{N}=21) \\
\text { Number, }(\%)\end{array}$ & p-Value \\
\hline Week 4 & $\begin{array}{l}\text { No } 7 / 21(33.3 \%) \text {, } \\
\text { Some-Tolerable } 14 / 21(66.7 \%)\end{array}$ & $\begin{array}{l}\text { No } 7 / 21(33.3 \%) \\
\text { Some-Tolerable } 14 / 21(66.7 \%)\end{array}$ & 0.628 \\
\hline Week 8 & $\begin{array}{l}\text { No } 12 / 21(57.4 \%) \\
\text { Some-Tolerable } 9 / 21(42.6 \%)\end{array}$ & $\begin{array}{l}\text { No } 14 / 21(66.7 \%) \\
\text { Some-Tolerable } 7 / 21(33.3 \%)\end{array}$ & 0.376 \\
\hline Week 12 & $\begin{array}{l}\text { No } 20 / 21(95.1 \%) \\
\text { Some-Tolerable } 1 / 21(4.9 \%)\end{array}$ & $\begin{array}{l}\text { No } 18 / 21(85.7 \%) \\
\text { Some-Tolerable } 4 / 21(14.3 \%)\end{array}$ & 0.756 \\
\hline
\end{tabular}

P-value: Fisher's Exact Test

Note: C: Control Treatment, HMC: Active Treatment, No: No reported skin reaction of the test product, Tolerable: Patients remain compliance with the local skin action of the test product

\section{DISCUSSION}

This study was an original investigation attempted to alleviate and delay progression of chronic venous disease (CVD) in clinical practice using herbal medicine as a combination with probable evidence from other herbal extract studies. There were possible herbal extracts which had been reported, such as Asiaticoside, Horse-Chestnut Seed Extract (HCSE), Extract of a French Maritime Pine Bark (Pycnogel ${ }^{\circ}$ ), Micronized Purified Flavonoid Fraction (MPFF), Ginkor Fort, Buckwheat Herb Tea, Red-Vine-Leaf Extract (RVLE), Chinese herbal medicine, and even Acetylsalicylic Acid (ASA $)^{21}$. There has been a lack of definitive conclusions in past studies due to limited study design and limited recruitment of patients and samples. Due to such limitations, in this study, we used the socalled Herbal Medicine Combination (HMC) to combine the potential beneficial effects of selected compounds (and their active ingredients) into one formulation. This innovative pharmaceutical method for encapsulation of Acetylsalicylic Acid with Beeswax was adopted to minimize the possible risk for oral administration.

For Asiaticoside, in a multicenter, double-blind (versus placebo) study of two-month oral administration, among 94 patients suffering from venous insufficiency of the lower limbs, it was reported that a significant difference $(\mathrm{p}<0.05)$ was seen in favor of Asiaticoside. There was observed improvement of symptoms of leg heavy and leg edema, and an overall satisfactory evaluation by the patients ${ }^{33}$. A prospective, randomized trial of an oral preparation of Asiaticoside among 40 venous microangiopathy patients with venous hypertension, either taking a 60 -mg tablet of TECA or placebo, twice daily for 6 weeks as a pre-andpost treatment comparison, demonstrated a decrease in the resting flux (29\%) and an improvement (increase) in venoarteriolar response (52\%). However, there was no controlled or direct comparison between placebo and active treatment groups ${ }^{34-37}$.

The other assessed effects of oral Asiaticoside (60-mg) tablets taken twice daily for 4 weeks, among 50 patients with ankle edema due to venous hypertension, 
before and after treatment, also showed a significant improvement with $34 \%$ reduction in the rate of ankle swelling, when compared with before treatment. This suggests that chronic venous insufficiency could start as early as the development of leg edema to control deterioration leading to leg ulcerations and it suggests that the complex actions and microcirculation are essential for reducing edema ${ }^{37}$.

This investigation study was conducted in mild to moderate CVD patients to overcome research gaps in other various studies by improvingthe design as a double-blind, randomized controlled trial and by adding a possible standard control (in this formulation, Acetylsalicylic Acid). This investigation minimized possible systematic risks of bias, thus allowing formild-to-moderate CVD patients to be selected based on diagnosis by a specialist surgeon. All assessments were performed by both the surgeon and a specially trained research nurse- for patient- reported outcome assessment and verification of data collection and validation. The overall allocation of treatment was blinded for the research nurse, surgeon, and patients- from the start of investigation (since all treatments were provided in a similarly sealed aluminum tube assigned only by the randomized numbers). This was the case for the two sets of treatments and throughout the trial period.

The overall primary objective or endpoint was the response to treatment (responder rate), defined as the reduction of $50 \%$ of Venous Clinical Severity Score after 4 weeks of treatment, when comparing the active (HMC) and the control (C) treatments. The overall responder rate for HMC treatment was $57.1 \%$, which was far better than that of the control (9.5\%). In addition, the lower responder rate $(9.5 \%)$ of the control group should warrant further investigation into whether the health outcomes response of the HMC reflected synergistic effects of $1 \%$ Acemannan and 2\% Asiaticoside, rendered by the $2 \%$ Acetylsalicylic Acid. This investigation, however, was limited in our knowledge whether 2\% Asiaticoside and 1\% Acemannan in the gel base could truly reabsorb through topical application, despite the fact that the $2 \%$ Acetylsalicylic acid in Beeswax encapsulation test reflected robust topical absorption (as tested in Franz Cell model). This investigation has limitations in that it only reflects mild-to-moderate symptoms of CVD patients. In spite of the mild degree of disease, the overall venous clinical severity observed at the early weeks (e.g. week 4) did not change at the end of the trial (week 12), though some degree of improvement was observed for the control group- from $9.5 \%$ to $19.4 \%$ (at 12 weeks).
The overall treatment impact on symptoms may possibly have limitations to some extent as the response to treatment remain unchanged after 12 weeks of follow-up. Further ad hoc analysis of benefits in specific leg symptoms from treatment demand a larger sample size for further investigation. However, ourcurrent evidence from the assessment ofboth patient symptom perception self-rated score and physicianrated score by venous clinical severity, the overall health-related quality of life was consistent. This investigation supports that there are possible attributes impacted by both Asiaticoside and Acemannan on the overall clinical and symptoms based on assessments. HMC could be considered an optional treatment for early chronic venous disease patients with leg symptoms. This investigation could be considered an early investigation for the possible implication of herbal medicine combination as well as improvement through pharmaceutical reformulation and innovation.

\section{CONCLUSIONS}

Herbal medicine combination containing 2\% Asiaticoside and 1\% Acemannan in 2\% Acetylsalicylic Acid in Beeswax encapsulation was based and developed as a topical administration; it provided significantly better response rate than the control base. The clinical improvement of symptoms assessed by venous clinical severity was consistently observed over the 12 weeks of administration and found to be similar to the assessment of patient self-rated symptom perception and patient self-rated health-related quality of life. The overall frequency of adverse events reported for the active treatment was not significantly different than that reported for the control treatment. Some limitations of the investigation remains to be improved in future studies; however, this investigation partially demonstrates the therapeutic beneficial impact of both Asiaticoside and Acemannan in combination, as an optional management strategy for mildto-moderate chronic venous disease. Further investigation demands a larger numbers of patients to decipher the impacts of specific leg symptoms.

Participants in this investigation may be perceived, though not yet definitively confirmed the diagnosis as CVD patients by the physician at the time of interviews could inevitably causing recall bias. The participants recruited in the hospital setting may expose the higher risk of recall bias for psychological components of the score or patients-center score as well as patient self-perception symptoms. This study was a pilot efficacy test of hypothesis which may demand 
larger sufficient sample size and in a different population, at various severity of CVD patients to support the further conclusion of the outcomes.

\section{ABBREVIATIONS}

ASA: Acetylsalicylic Acid, Aspirin

C: Control

CEAP: Comprehensive Classification System for Chronic Venous Disorders (CEAP), the CEAP classification include a description of the clinical class (C) based upon objective signs, the etiology (E), the anatomical (A) distribution of reflux and obstruction in the superficial, deep and perforating veins, and the underlying pathophysiology $(\mathrm{P})$, whether due to reflux or obstruction.

CVD: Chronic Venous Disease

HCSE: Horse-Chestnut Seed Extracts

HMC: Herbal Medicine Combination

MMPF: Micronized Purified Flavonoid Fraction

MOS CIVIQ14: the Medical Outcomes Study 14item Short Form Health Survey for Chronic Venous Insufficiency

PSSS: Patient Self-Rated Symptoms Score based on Visual Analog Scale

RVLE: Red-Vine-Leaf Extract

TECA: Titrated Extracts Centella Asiatica

VCSS: Venous Clinical Severity Score

\section{COMPETING INTERESTS}

We declare that there are no competing financial interests in this manuscript, from any person or organization, and no conflict of interest involved in the outcomes or findings.

\section{AUTHORS' CONTRIBUTIONS}

AU: conceived the study, designed the protocol, trained the research associates, monitored the data collection, analyzed the data, and wrote the first manuscript draft.

NK: conceived the study, designed the protocol, obtained research funds, edited the manuscript, and advised on the entire research paper.

PP: conceived the study, supervised the research associates, recruited participants, and validated data and the manuscript.

All authors read and approved the final manuscript.

\section{AVAILABILITY OF DATA AND MATERIALS}

The datasets generated and analyzed during the current study are part of the full dataset of the registered intervention trial and shall be available from the corresponding author upon reasonable request.

\section{ACKNOWLEDGMENTS}

We would like to thank all research associates:Onnapa Yodkham (B..S RN.) and Peerapich Kaewlung (B... RN) at Chiangraiprachanukroh Hospital Chiang Rai, Thailand for assistance in our research. We would like to thank members and the Dean of the Faculty of Pharmacy at Eastern Asia University, Bangkok Thailand, Associate Professor Papavadee Klongpitayaphong, MSc (Columbia University, NY, USA), Assistant Professor Vichien Thanindratarand, MPharm (Chulalongkorn University), and Associate Professor Nongluks Sriubolmas, PhD (Mahidol University), for their support in the formulation development of the HMC employed in this study.

\section{REFERENCES}

1. Onida S, Davies AH. Predicted burden of venous disease. Phlebology. 2016;31(1):74-9. 26916773. Available from: 10.1177/ 0268355516628359 .

2. Nicolaides $\mathrm{AN}$, Cardiovascular DE, Research $\mathrm{T}$, of Vascular Surgery European S, The IASACO, of Angiology International $U$, et al. Investigation of chronic venous insufficiency: A consensus statement (France, March 5-9, 1997). Circulation. 2000;102(20):E126-63. 11076834. Available from: 10.1161/01. CIR.102.20.e126.

3. Rakprasit J. A study of factors associated with varicose veins among women workers in the electronic factories. Bangkok: Mahidol University; 2008. Available from: http://mulinet11.li.mahidol.ac.th/thesis/2551/cd417/4836068. pdf(accessedDecember20,2018).

4. Wongkongkam K. Factors affecting the quality of life among venous leg ulcer patients with compression bandaging. Bangkok: Mahidol University; 2009.

5. Kanchanabat B, Wongmahisorn Y, Stapanavatr W, Kanchanasuttirak $\mathrm{P}$, Manomaiphiboon $\mathrm{A}$. Clinical presentation and patterns of venous reflux in Thai patients with chronic venous insufficiency (CVI). Eur J Vasc Endovasc Surg. 2010;40(3):399402. 20561800. Available from: 10.1016/j.ejvs.2010.04.017.

6. Callejas JM, Manasanch J, ETIC G. Epidemiology of chronic venous insufficiency of the lower limbs in the primary care setting. Int Angiol. 2004;23(2):154-63. 15507894.

7. Wolinsky CD, Waldorf H. Chronic venous disease. Med Clin North Am. 2009;93(6):1333-46. 19932334. Available from: 10 1016/j.mcna.2009.08.001.

8. Akbulut B, Uçar HI, Oç M, Ikizler M, Yorgancioglu C, Dernek S, et al. Characteristics of venous insufficiency in western Turkey: VEYT-I study. Phlebology. 2012;27(7):374-7. 22316598. Available from: 10.1258/phleb.2011.011100.

9. Sierra-García GD, Castro-Ríos R, González-Horta A, Lara-Arias J, Chávez-Montes A. Acemannan, an extracted polysaccharide from Aloe vera: A literature review. Nat Prod Commun 2014;9(8):1217-21. 25233608.

10. http://apps.who.int/medicinedocs/en/d/Js2200e/6.html

11. Puataweepong $P$, Dhanachat $M$, Dangprasert $S$, Sithatani $C$, Sawangsilp T, Narkwong L, et al. The efficacy of oral Aloe vera juice for radiation induced mucositis in head and neck cancer patients: a double-blind placebo-controlled study. Asian Biomed. 2009;3(4):375-82.

12. Jr JEF. The stimulation of postdermabrasion wound healing with stabilized aloe vera gel-polyethylene oxide dressing. J Dermatol Surg Oncol. 1990;16(5):460-7. 2341661. Available from: 10.1111/j.1524-4725.1990.tb00065.x. 
13. Syed TA, Ahmad SA, Holt AH, Ahmad SA, Ahmad SH, Afzal $M$. Management of psoriasis with Aloe vera extract in a hydrophilic cream: a placebo-controlled, double-blind study. Trop Med Int Health. 1996;1(4):505-9. 8765459. Available from: 10.1046/j.1365-3156.1996.d01-91.x.

14. Vardy DA, Cohen AD, Tchetov T, Medvedovsky E, Biton A. A double-blind, placebo-controlled trial of an Aloe vera (A. barbadensis) emulsion in the treatment of seborrheic dermatitis. J Dermatolog Treat. 1999;10(1):7-11. Available from: 10.3109/09546639909055904.

15. Blitz JJ, Smith JW, Gerard JR. Aloe vera gel in peptic ulcer therapy: preliminary report. J Am Osteopath Assoc. 1963;62:7315. 13971654.

16. Davis K, Philpott S, Kumar D, Mendall M. Randomised doubleblind placebo-controlled trial of aloe vera for irritable bowel syndrome. Int J Clin Pract. 2006;60(9):1080-6. 16749917. Available from: 10.1111/j.1742-1241.2006.00980.x.

17. Gohil KJ, Patel JA, Gajjar AK. Pharmacological Review on Centella asiatica: A Potential Herbal Cure-all. Indian J Pharm Sci. 2010;72(5):546-56. 21694984. Available from: 10.4103/0250$474 X .78519$

18. Bylka W, Kornobis J. [Butcher's Broom, in the treatment of venous insufficiency]. Pol Merkur Lekarski. 2005;19(110):234-6. 16245442.

19. European Medicines Agency, Assessment report on Centella asiatica (L.) Urban, herbal; 2018. Available from: http:// www.ema.europa.eu/docs/en_GB/document_library/Herbal_ -_HMPC_assessment_report/2012/06/WC500128144.pdf.

20. Kiesewetter H, Koscielny J, Kalus U, Vix JM, Peil H, Petrini O, et al. Efficacy of orally administered extract of red vine leaf AS 195 (folia vitis viniferae) in chronic venous insufficiency (stages I-II). A randomized, double-blind, placebo-controlled trial. Arzneimittelforschung. 2000;50(2):109-17. 10719612.

21. Ernst $\mathrm{E}$. Herbal medications for common ailments in the elderly. Drugs Aging. 1999;15(6):423-8. 10641953. Available from: 10.2165/00002512-199915060-00002.

22. Rohdewald P. A review of the French maritime pine bark extract (Pycnogenol), a herbal medication with a diverse clinical pharmacology. Int J Clin Pharmacol Ther. 2002;40(4):158-68. 11996210. Available from: 10.5414/CPP40158.

23. Lim KS, Tang MB, Goon AT, Leow YH. The role of topical traditional chinese medicaments as contact sensitisers in chronic venous leg ulcer patients. Ann Acad Med Singapore. 2007;36(11):942-6. 18071606.

24. Felixsson E, Persson IA, Eriksson AC, Persson K. Horse chestnut extract contracts bovine vessels and affects human platelet aggregation through 5-HT(2A) receptors: an in vitro study. Phytother Res. 2010;24(9):1297-301. 20148408. Available from: $10.1002 /$ ptr.3103.

25. Pawlaczyk I, Czerchawski L, Kańska J, Bijak J, Capek P, Pliszczak-Król A, et al. An acidic glycoconjugate from Lythrum salicaria L. with controversial effects on haemostasis. Journal of ethnopharmacology. 2010;131(1):63-69.

26. Kirsch I, Weixel LJ. Double-blind versus deceptive administration of a placebo. Behav Neurosci. 1988;102(2):319-23. 3365327. Available from: 10.1037/0735-7044.102.2.319.

27. Schoonees A, Visser J, Musekiwa A, Volmink J. Pycnogenol( $\left.{ }^{\oplus}\right)$ for the treatment of chronic disorders; 2012. Available from: 10.1002/14651858.CD008294.

28. Morling JR, Yeoh SE, Kolbach DN. Rutosides for treatment of post-thrombotic syndrome. Cochrane Database Syst Rev. 2013;p. CD005625. 23633330. Available from: 10.1002/ 14651858.CD005625.pub2.

29. Marderosian AD, Beutler JA. Aloe in The Review of Natural Products, the Most Complete Source of Natural Product Information. Wolters-Kluwer Health; 2012.
30. Yongchaiyudha S, Rungpitarangsi V, Bunyapraphatsara N, Chokechaijaroenporn O. Antidiabetic activity of Aloe vera $\mathrm{L}$. juice. I. Clinical trial in new cases of diabetes mellitus. Phytomedicine. 1996;3(3):241-3. 23195077. Available from: 10 . 1016/S0944-7113(96)80060-2.

31. Brinkhaus B, Lindner M, Schuppan D, Hahn EG. Chemical pharmacological and clinical profile of the East Asian medica plant Centella aslatica. Phytomedicine. 2000;7(5):427-448.

32. Arora D, Kumar M, Dubey SD. Centella asiatica-A Review of it's Medicinal Uses and Pharmacological Effects. Journal of Natural remedies. 2002;2(2):143-149.

33. Pointel JP, Boccalon $\mathrm{H}$, Cloarec $M$, Ledevehat $C$, Joubert $M$. Titrated extract of Centella asiatica (TECA) in the treatment of venous insufficiency of the lower limbs. Angiology. 1987;38(1 Pt 1):46-50. 3544968. Available from: 10.1177/ 000331978703800106.

34. Cesarone MR, Belcaro G, Rulo A, Griffin M, Ricci A, Ippolito E, et al. Microcirculatory effects of total triterpenic fraction of Centella asiatica in chronic venous hypertension: measurement by laser Doppler, TcPO2-CO2, and leg volumetry. Angiology. 2001;52(2_suppl):S45-8. 11666123. Available from: 10.1177/000331970105202S09.

35. Cesarone MR, Laurora G, Sanctis MTD, Incandela L, Grimaldi R, Marelli $C$, et al. [The microcirculatory activity of Centella asiatica in venous insufficiency. A double-blind study]. Minerva Cardioangiol. 1994;42(6):299-304. 7936334.

36. Belcaro G, Rulo A, Cesarone MR, Sanctis MTD, Incandela L, Griffin $M$, et al. Capillary filtration in venous hypertension: evaluation with the vacuum suction chamber device and straingauge plethysmography. Angiology. 2001;52(2_suppl):S3943. 11666122. Available from: $10.1177 / 000331970105202 S 08$.

37. Chong NJ, Aziz Z. A systematic review of the efficacy of Centella asiatica for improvement of the signs and symptoms of chronic venous insufficiency. Evidence-Based Complementary and Alternative Medicine. 2013;2013.

38. Rutherford RB, Jr FTP, Comerota AJ, Kistner RL, Meissner MH, Moneta GL. Venous severity scoring: an adjunct to venous outcome assessment. J Vasc Surg. 2000;31(6):1307-12. 10842165. Available from: $10.1067 / \mathrm{mva} .2000 .107094$.

39. Meissner MH, Natiello C, Nicholls SC. Performance charac teristics of the venous clinical severity score. J Vasc Surg. 2002;36(5):889-95. 12422097. Available from: 10.1067/mva. 2002.128637.

40. Eklöf B, Rutherford RB, Bergan JJ, Carpentier PH, Gloviczki $\mathrm{P}$, Kistner RL, et al. Revision of the CEAP classification for chronic venous disorders: consensus statement. J Vasc Surg. 2004;40(6):1248-52. 15622385. Available from: 10.1016/j.jvs 2004.09.027.

41. Talley NJ, Zinsmeister AR, Schleck CD, 3rd LJM. Dyspepsia and dyspepsia subgroups: a population-based study. Gastroenterology. 1992;102(4 Pt 1):1259-68. 1551533. Available from: 10.1016/0016-5085(92)90764-P.

42. Launois R, Reboul-Marty J, Henry B. Construction and validation of a quality of life questionnaire in chronic lower limb venous insufficiency (CIVIQ). Qual Life Res. 1996;5(6):539-54 8993100. Available from: 10.1007/BF00439228.

43. Moine JGL, Fiestas-Navarrete L, Katumba K, Launois R. Psychometric Validation of the 14 items Chronlc Venous Insufficiency Quality of Life Questionnaire (CIVIQ-14): Confirmatory Factor Analysis. Eur J Vasc Endovasc Surg. 2016;51(2):268-74. 26520178. Available from: 10.1016/j.ejvs.2015.08.020.

44. Udombhornprabha A, Kanchanakhan N, Phongmanjit P. A Short-term Safety Assessment of Herbal Medicine Combination: A Randomized Cross-over Controlled Trial in Healthy Volunteer, EAU Heritage. J Sci Technol. 2017;11(3):72-84. 